\title{
Cardiac emergencies: Blunt chest trauma
}

\author{
George Karatasakis, MD, FESC \\ Onassis Cardiac Surgery Center, Athens, Greece
}

Abstract

Blunt chest trauma is considered a major health problem worldwide because of the tremendous increase of the motor vehicle accidents. Any part of the heart or the great vessels can be injured. Hemopericardium and myocardial contusion are the most frequent cardiac lesions in patients who survive a motor vehicle accident. Rupture of a cardiac chamber, the aorta, or the coronary arteries is often fatal. Valve ruptures especially of the tricuspid valve carry a better prognosis. Diagnosis is based on troponin and cardiac enzymes measurement, ECG changes, chest X-ray, echocardiography and spiral computed tomography. Management of patients with compromised hemodynamics and progressive deterioration is surgical often on an emergent basis.

Key words blunt chest trauma, heart and great vessel injury

C hest injury may affect any organ situated in the thoracic cavity including the heart and great vessels. Blunt mechanisms are more often involved in chest wounds while penetrating traumas are less frequent.

Injuries of the skeletal components of the chest (pectoral muscles, ribs, clavicles etc.) have a better prognosis, provided that the broken bones do not penetrate any vital organ. Routine clinical and laboratory evaluation, leads to diagnosis in the majority of cases.

Conversely, injuries of the heart or the great vessels represent a diagnostic challenge, because rapid and precise diagnosis is required especially in patients with hemodynamic instability. Significant heart or great vessels damage is related to compromised prognosis. Blunt trauma that causes cardiac or aortic rupture usually leads to death because of massive hemorrhage or cardiogenic shock before the patient reaches the hospital.

\section{Etiology-Frequency}

The most common cause of blunt chest trauma in modern world is motor vehicle accident which accounts for up to $80 \%$ of such injuries ${ }^{1}$.

The widespread use of air bags together with the steady increase of car accidents over the last 60 years (almost tenfold between 1950-1990) attributed to the establishment of blunt chest trauma as a major health problem worldwide. It has been estimated that thoracic injury occurs in 12 persons per million population per year.

Interestingly there is an impressive difference between clinical and autopsy data concerning cardiac injuries. Clinical reports, on injuries of specific anatomic parts of the heart are rare in the literature and in some instances only case reports can be found. Conversely autopsy studies demonstrate that the heart is injured in $20 \%$ of patients dying as a consequence of a traffic accident. Fur- thermore, thoracic aorta damage is involved in $15 \%$ of patients dying because of motor vehicle accidents ${ }^{2}$. This discrepancy, between clinical and autopsy findings, may lead to the conclusion that the majority of severe injuries of the heart and great vessels remain undiagnosed with lethal consequences. Rupture of a cardiac chamber, is encountered in $35-65 \%$ of autopsies, of patients dying following a cardiac trauma and only in $0.3-0.9 \%$ in clinical series $^{3}$. This dramatic difference could be decreased with early clinical suspicion of cardiac trauma in motor vehicle accident victims.

Virtually all anatomic components of the heart can be damaged.

1. The coronary arteries may present with laceration, rupture, dissection or acute thrombosis.

2. The ventricular myocardium may present ruptures (free wall or intraventricular septum) contusion or aneurysms.

3. Valvular lesions include leaflet or chordal rupture and papillary muscle dysfunction because of myocardial contusion.

4. Injury of the great vessels including rupture, dissection, aneurysm formation, or thrombotic occlusion (5).

5. Pericardial injury including hemopericardium, tamponade, or -in the long term-constrictive pericarditis.

6. Commotio cordis

\section{Pathophysiology}

A sudden violent impact of the chest on a broad surface like an inflated airbag may lead to an enormously high and sudden rise of the intracardiac pressure and produce a tear to a ventricular or atrial wall ${ }^{3}$.

Furthermore abrupt deceleration which often occurs in motor vehicle accidents produces cardiac ruptures in specific sites, where great vessels are entering the heart. The 
aortic annulus is a characteristic site of rupture in motor vehicle accidents with abrupt deceleration. The inerzia of the heart differs from the inerzia of the ascending aorta, therefore the force applied to the heart is greater than the force applied to the ascending aorta. Because of this difference the heart moves forward faster than the aorta during abrupt deceleration leading to detachment of the heart from the aorta at the site of the annulus. Similar forces are applied at the aortic ligament and the aortic isthmus ${ }^{6-8}$.

Another common consequence of blunt chest trauma is myocardial contusion which occurs as a result of impact and compression of the myocardium on the chest wall. It is characterized by localized hemorrhagic areas situated within the normal myocardium. Areas of necrosis may also coexist. Clinical evidence of contusion can be rhythm disturbances or conduction delays. Myocardial contusion should be differentiated from myocardial infarction which is usually the consequence of coronary artery dissection or rupture also complicating blunt chest trauma. These two entities differ in clinical severity, prognosis and natural history. In myocardial contusion regional wall motion abnormalities are not indicative of a specific coronary artery territory. Conversely myocardial infarction due to post-traumatic coronary artery laceration has a classical distribution of regional wall motion abnormalities. Furthermore myocardial contusion is a transient phenomenon, resolving usually a few weeks after the cardiac injury ${ }^{9,10}$.

\section{Diagnosis of heart and great vessels injury in patients with blunt chest trauma}

The diagnosis of cardiac injury in patients with blunt chest trauma cannot be based solely on clinical findings, because these patients, often victims of motor vehicle accidents, present with a variety of symptoms and signs related to the accident.

Obviously all patients with blunt chest trauma would complain for chest pain- when conscious- and are usually tachypnoic and tachycardic, because of the pain and the psychological stress. Evidence of cardiogenic shock can raise the suspicion of cardiac damage. Chest x-rays may show evidence of widened cardiac silhouette which is not a specific sign. However enlargement of the upper mediastinum is often related to arterial injury.

Measurement of cardiac enzymes and troponin I level is often useful, although not entirely specific, in diagnosing cardiac necrosis following coronary artery rupture or dissection because of trauma. Troponin levels correlate with two - dimensional echocardiographic and ECG findings in patients with post-traumatic myocardial infarction. However these indices are not sensitive enough in cardiac contusion and cannot be used to differentiate contusion from infarction. Elevated troponin I can be found in more than half of patients with blunt chest trauma. In $60 \%$ of patients with troponin I $>1 \mathrm{ng} / \mathrm{ml}$ echocardiography reveals regional wall motion abnormalities ${ }^{11}$.

Twelve lead ECG should be performed in all patients with blunt chest trauma. In cases of myocardial injury it may reveal evidence of necrosis or conduction disturbances, but its diagnostic value has not been proven ${ }^{12}$.

Holter monitoring may reveal delayed evidence of rhythm or conduction disturbances in initially stable patients.
Thansthoracic echocardiography should be performed in patients exhibiting haemodynamic instability, shortness of breath, hypoventilation or complex arrhythmias. The development of pericardial effusion or tamponade can be readily diagnosed by transthoracic echocardiography. Valvular lesions include tears of valve leaflet, affecting more often the tricuspid valve because it is located in the vicinity of the anterior chest wall and lead to significant tricuspid regurgitation. This condition may occur clinically silent and reveal in a remote time period. Conversely tears of the aortic cusps usually produce severe aortic regurgitation and are often combined with aortic wall injuries or aortic dissection ${ }^{13}$.

An interesting point is that feasibility of transesophageal echocardiography in patients with blunt chest trauma is greater than feasibility of tranthoracic echocardiography in such patients. In a study of 134 patents, feasibility of transthoracic echocardiography was only $38 \%$ while transesophageal echocardiography was feasible in $98 \%$ of patients ${ }^{14}$. In this particular clinical study, myocardial contusion and pericardial effusion were the two most frequent cardiac lesions complicating blunt chest trauma. Myocardial contusion and pericardial effusion were found to be very common yet in another study which included 117 patients with blunt chest trauma ${ }^{15}$. Using transesophageal echocardiography, ECG and CK MB levels, authors of that study reported that although patients with cardiac lesion had higher incidence of ECG and CKMB abnormalities, these abnormalities were not enough to depict all patents with cardiac injuries evident on transesophageal echocardiography.

Spiral computed tomography is important in patients with blunt chest trauma, especially those with suspected arterial injury ${ }^{16}$. Sensitivity of spiral computed tomography for arterial injuries is $73 \%$ and specificity $100 \%$. For lesions of the descending thoracic aorta and its branches, computed tomography is of higher diagnostic value than transesophageal echocardiography ${ }^{17}$.

Chest x-ray, ECG, cardiac enzymes and troponin I should be obtained in all patients with blunt chest trauma. Those with ischemic changes on ECG, significant elevation of the biomarkers, complex arrhythmias, hemodynamic instability, or persisting and progressive shortness of breath should be placed in intensive care unit and proceed immediately with transthoracic or transesophageal echocardiography and spiral computed tomography. For the rest, a 24 hours cardiac monitoring should suffice.

\section{Management}

Given the complexity of the lesions and the mechanisms involved treatment should be individualized. Patients with hemopericardium that impairs cardiac filling or hemothorax and clinical instability should undergo surgical drainage. Needle pericardiocentesis is not recommended - unless surgery is unavailable- because abrupt fall of the intrapericardial pressure may produce massive exsanguination and death, if a cardiac cavity rupture has occurred. Patients with dissection or rupture of a coronary artery, extensive myocardial infarction and cardiogenic shock should be managed by placing an intraortic balloon pump. Percutaneous revascularization is not always effective and coronary artery by pass grafting should 
be performed. Valvular lesions especially of the aorta often need emergent surgery ${ }^{18}$. Conversely patients with mitral valve lesions causing mitral regurgitation usually remain stable for weeks.

\section{Commotio cordis}

Commotio cordis represents a specific type of blunt chest trauma produced by a strong precordial impact when a hard object of small size (a hockey puck) hits the chest. In cases that this impact coincides with the upstroke of the T wave it may produce a rapid increase of intracardiac pressure and lead to ventricular fibrillation ${ }^{19}$.

\section{Conclusion}

The heart and the great vessels can be severely damaged in victims of motor vehicle accidents. The high incidence of such accidents underlines the importance of blunt chest trauma. The diagnosis is based on biomarkers measurements, ECG, and imaging techniques. The most important factor to make a rapid and precise diagnosis and improve dramatically patients' prognosis is physician's alertness in the management of blunt chest trauma.

\section{References}

1. Bu'Lock FA, Prothero A, Shaw C, et al. Cardiac involvement in seatbelt-related and direct sternal trauma: a prospective study and management implications. Eur Heart J 1994;15:1621-7.

2. Parmley LF, Manion WC, Mattingly TW. Nonpenetrating traumatic injury of the heart. Circulation 1958;18:371-96.

3. Pretre R, Chilcott M. Blunt trauma to the heart and great vessels. N Engl J Med 1997;336:626-32.

4. Shorr RM, Crittenden M, Indeck M, Hartunian SL, Rodriguez A. Blunt thoracic trauma: analysis of 515 patients. Ann Surg 1987;206:200-5.

5. Prerre R, LaHarpe R, Cheretakis K, et al. Blunt injury to the ascending aorta: three patterns of presentation. Surgery 1996;119:603-10.
6. Mattox KL. Approaches to trauma involving the major vessels of the thorax. Surg Clin North Am 1989;69:77-91.

7. von Oppell UO, Dunne TT, De Groot MK, Zilla P. Traumatic aortic rupture: twenty-year metaanalysis of mortality and risk of paraplegia. Ann Thorac Surg 1994;58:585-93.

8. Kram HB, Wohlmuth DA, Appel PI, Shoemaker WC. Clinical and radiographic indications for aortography in blunt chest trauma. J Vasc Surg 1987;6:168-76.

9. Cachecho R, Grindlinger GA, Lee VW. The clinical significance of myocardial contusion. J Trauma 1992;33:68-73.

10. BiffI WL, Moore FA, Moore EE, Sauaia A, Read RA, Burch JM. Cardiac enzymes are irrelevant in the patient with suspected myocardial contusion. Am J Surg 1994;168:523-8.

11. Mori F, Zuppiroli A, Ognibene A, et al. Cardiac contusion in blunt chest trauma: a combined study of transesophageal echocardiography and cardiac troponin I determination. Ital Heart J 2001;2:222-7.

12. Dubrow TJ, Mihalka J, Eisenhauer DM, et al. Myocardial contusion in the stable patient: what level of care is appropriate? Surgery 1989;106:267-74.

13. Raptopoulos V, Sheiman RG, Phillips DA, Davidoff A, Silva WE. Traumatic aortic tear: screening with chest CT. Radiology 1992;182:667-73.

14. Chirillo F, Totis O, Cavarzerani A et al. Usefulness of transthoracic and transoesophageal echocardiography in recognition and management of cardiovascular injuries after blunt chest trauma. Heart 1996;75:301-6.

15. Garcia-Fernandez MA, Lopez-Perez JM, Perez-Castellano N. Role of transesophageal echocardiography in the assessment of patients with blunt chest trauma: correlation of echocardiographic findings with the electrocardiogram and creatine kinase monoclonal antibody measurements. Am Heart J 1998;135:476-81.

16. Catoire $P$, Orliaguet $G$, Lin N, et al. Systematic transesophageal echocardiography for detection of mediastinal lesions in patients with multiple injuries. J Trauma 1995;38:96-102.

17. Vignon $P$, Boncoeur MP, Francois $B$ et al. Comparison of multiplane transesophageal echocardiography and contrast-enhanced helical $\mathrm{CT}$ in the diagnosis of blunt traumatic cardiovascular injuries. Anesthesiology 2001;94:615-22.

18. Pretre R, Faidutti B. Surgical management of aortic valve injury following nonpenetrating trauma. Ann Thorac Surg 1993;56:1426-31.

19. Maron BJ, Estes NA $3^{\text {rd }}$. Commotio cordis. N Engl J Med 2010;362:917-27. 